\title{
Active MMW/Terahertz Security System Based on Bessel Beams
}

\author{
Igor Minin and Oleg Minin \\ Siberian State Geodesial Academy, Plahotnogo 10, Novosibirsk 630127, Russia \\ Correspondence should be addressed to Igor Minin; prof.minin@gmail.com
}

Received 9 April 2013; Accepted 8 May 2013

Academic Editors: S. Gallego and G. S. Maciel

Copyright ( 2013 I. Minin and O. Minin. This is an open access article distributed under the Creative Commons Attribution License, which permits unrestricted use, distribution, and reproduction in any medium, provided the original work is properly cited.

\begin{abstract}
The novel concept of the security system based on THz Bessel beams is offered. The system is based on a novel THz diffractive optics for scanning the person (without the application of $\mathrm{THz}$ laser) and on a sensitive scheme for the detection of the reflected and scattered THz radiation. The development of enabling technology, namely, sensitive detector arrays and Millimeter wave/THz diffractive optics, will allow building compact, easy-to-use millimeter wave/THz imaging systems without expensive cost THz laser. The scanning properties of diffractive optics for Bessel beam are investigated.
\end{abstract}

\section{Introduction}

The novel technology of a new class of imaging systems for detecting threats, explosives, pathogens, and chemicals hidden by a person or inside an object such as letters or luggage is based on active imaging concepts implementing terahertz $(\mathrm{THz})$ or/and millimeter wave (MMW) radiation. In combination with advanced sensors based on diffractive optics with Bessel beams this will lead to an increased level of security at public places, for example, airports or private places as a room.

\section{The Principles of Security Systems}

As well known [1] there are three essential components to a laser security system: a laser, a detector, and sensing electronics. The laser is a concentrated light source that puts out a straight line "pencil beam" of light (or IR) of a single color. The detector is sensitive to light and puts out a voltage when the laser light hits it. The detector is connected to the sensing electronics. When the laser beam is interrupted and cannot reach the detector, its voltage output changes, and the electronics sense the change and put out a warning signal.

Usually you want to protect the perimeter of a room, or at least the entrances. So laser security systems start with a laser pointing to a small mirror. The first mirror is angled to direct the beam to a second small mirror, and so on until the final mirror directs the beam to the detector. If the beam is interrupted anywhere between the laser and the detector, the electronics will put out the warning signal.

Disadvantages of laser security systems are as follows.

(1) The laser beam is visible by eye or special (e.g., nightvision) systems.

(2) The laser beam is visible in smoke.

(3) The work of laser security system can be destroyed by high-absorption smoke and so forth.

But a THz/MMW waves are less sensitive to smoke and invisible by eyes. For example, under identical dust concentrations, the IR signal at $1.5 \mu \mathrm{m}$ wavelength is significantly more attenuated than the $\mathrm{THz}$ signal $(625 \mathrm{GHz})$, which shows almost no degradation from the channel perturbation $[2,3]$.

There has been much interest in recent years in the properties of "diffractionless" beams, solutions to the wave equation that produce a line focus with invariant spot diameter over an extended depth of field. These solutions are known as Bessel beams which are propagation-invariant solutions of the wave equation since the transverse intensity profile, within the depth of field, is a Bessel function in the radial coordinate [4]. A Bessel beam can be formed by the interference of wave fronts propagating at an angle to the optic axis, an effect which can be achieved in a number of different ways. The mathematical representation of a Bessel beam can be interpreted as a superposition of plane waves, all with 


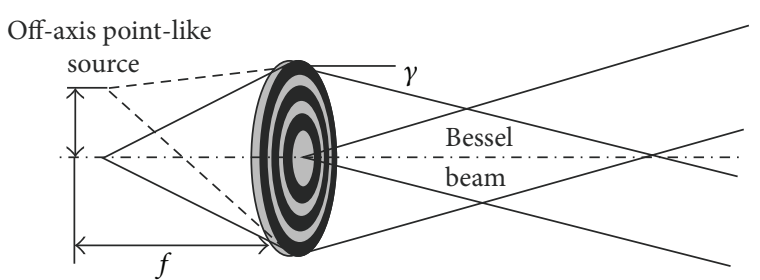

FIGURE 1: The generation of a Bessel beam using diffractive "lensplus-axicon" lens.

the same inclination angle $\gamma$ relative to the $z$-axis, but the azimuthal angles are ranging from 0 to $2 \pi$.

One popular method is to use a diffractive axicon [5] for a flat incident wave front usually in optic waveband. For the use in different quasioptical systems, or analog laser security system in $\mathrm{THz}$, elements are of interests that are obliquely illuminated from point-like source of radiation. Bessel beams in $\mathrm{mm}$ and submm wave regimes facilitate generating highly localized wave fields, which can penetrate inside bulk material.

For the purposes described above we consider a spherical wave front from point-like source of radiation, which is incident on a diffractive flat surface and represents an axicon lens. Such a wave front is converted to a converging conical wave front, which approaches the optic axis at an angle of $\gamma$ (Figure 1). The direction of the wave propagation extends an angle $\gamma$ with respect to the axis of symmetry, leading to a cylindrically symmetric wave pattern. The radial distribution of the incident wave front is mapped onto the optic axis, defining the depth of field of the line focus [6].

\section{Methods of Computation of the Fresnel-Kirchhoff Diffraction Integral}

Diffraction of a scalar wave by a diffractional element can be described mathematically using the Fresnel-Kirchhoff integral that in the general case has the form [7]:

$$
U\left(P_{0}\right)=\frac{A}{i \lambda} \iint_{S} \frac{\exp \left(-i k\left(r_{21}+r_{01}\right)\right)}{r_{21} r_{01}} \chi(r) d S,
$$

where $P_{0}$ is the observation point, $r_{01}, r_{21}$ are the distances from a point source to a certain point within the aperture and to the point source of emission, respectively, $\chi(r)$ is the inclination factor which is given by the Kirchhoff theory as

$$
\chi(r)=\frac{1}{2\left\{\cos \left(\vec{n}, \vec{r}_{01}\right)-\cos \left(\vec{n}, \vec{r}_{21}\right)\right\}},
$$

where $\vec{n}$ is the normal to the screen surface. As the divergent spherical wave transforms itself to the plane wave, the factor $\chi(r)$ tends to $K_{n}$. (Notes: In scalar theory of diffraction the amplitude of oscillations at $P$ is proportional to the size of the element $d S$ and also depends on the angle $\varphi$ between the normal $n$ to the zone and its radius $R_{n}$. This factor is taken into account by the coefficient $K_{n}$ ).

A universal and efficient method of computing integral (1) was considered in $[8,9]$ in the case of problems of diffraction

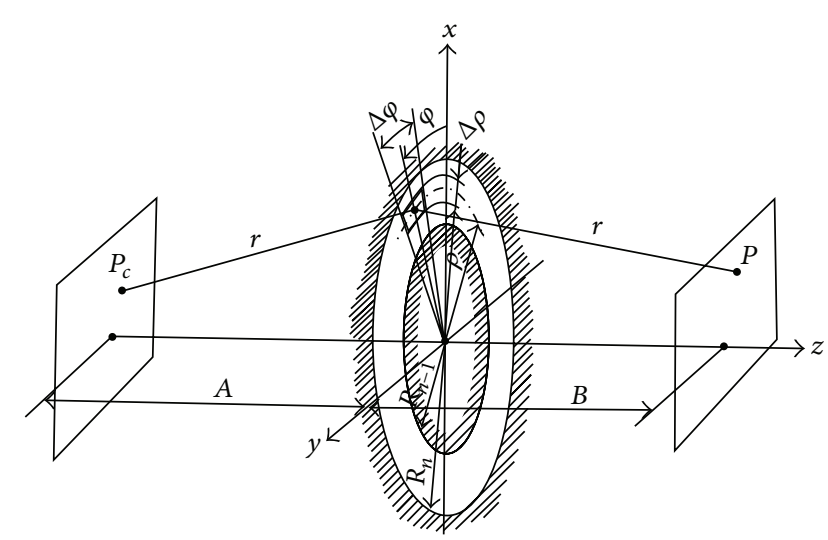

Figure 2: Diagram for computation of a diffraction element [8].

of scalar waves on diffractional elements. Let us consider, in correspondence with the symmetry of the problem, a cylindrical coordinate system $(\rho, \varphi, z)$; we place its origin over the centre of the diffraction segment. The axis $\mathrm{O} z$ is normal to diffractive element Figure 2 and the point $\mathrm{O}$ is in the centre of diffractive element.

Assume now that $l$ point-like emitters are placed in the region of space $Z<0$ at points with coordinates $P_{l}^{i}$. In the region $Z>0$ the field $U(P)$ diffracted by the diffraction element is determined at the observation point $P(\rho, \varphi, z)$. To simplify the presentation, we limit the case to a single emission source $(l=1)$ and a single Fresnel zone $n$ between the radii $R_{n-1}$ and $R_{n}$; we also use the rectangle method, which is acceptable from the point of view of computation accuracy of (1) and can now rewrite the initial integral (1) in the form:

$$
\sum_{m=1}^{M} \Delta S_{m} f_{m} \exp \left(i 2 \pi \psi_{m}\right)
$$

where $f_{m}, \psi_{m}$ are functions of $\left(\rho_{m}, \varphi_{m}\right)$, that is, functions of polar coordinates of the central point of the subarea $\Delta S_{m}=(\rho \Delta \rho \Delta \varphi)_{m}$. This shows, among other things, that for the integration step $\delta \approx \lambda / 4$ the number of terms in the sum (3) is $M \approx 2 \pi(D / \lambda)^{2}$ and a direct computation of (3) requires a considerable amount of computer time. To reduce the computation time (the number $M$ ) we resort to an obvious identity:

$$
\exp (i 2 \pi \psi)=(i 2 \pi \operatorname{frac}(\psi)) \quad(\psi>0),
$$

where $\operatorname{frac}(\psi)$ is the fractional part of the real function $\psi$. Hence, this function can always be reduced to the interval $[0,1)$. On this interval, we can introduce a discrete grid whose nodes are enumerated by an integral index:

$$
t=\operatorname{entier}(\psi T+0.5), \quad(0<\psi \leq 1, t=1,2 \ldots, T),
$$

where entier $(x)$ is the largest integer not exceeding $x$. Let $\psi$ assume on this interval one of $t \in[1, T]$ possible discrete values of $\psi_{t}$ that differ among themselves by $\Delta \psi=1 / T$. Then the phase $2 \pi \psi$ and the exponential term in (3) assume not more than $T$ different values. If we prescribe integration steps 
$\Delta \rho=\sigma$ and $\Delta \varphi=\sigma / \rho$, then the number of terms in the sum (3) at $T \approx D / \lambda$ is $M \approx 2 \pi T^{2}$. Therefore, for each $t$ we can form groups of terms of the type:

$$
\Delta S_{k t} f_{k t} \exp \left(i 2 \pi \psi_{t}\right), \quad\left(k=0,1, \ldots, k_{t}\right),
$$

where $k_{t}$ is found by counting the number of times that $\psi$ falls within the interval $t$ as $\left(\rho_{m}, \varphi_{m}\right)$ runs through the entire integration range. Then we find

$$
g\left(\psi_{t}\right)=\sum_{k=1}^{k t} \Delta S_{k t} f_{k t}, \quad t=1,2, \ldots, T
$$

and finally the sum

$$
\sum_{t=1}^{T} g\left(\psi_{t}\right) \exp \left(i 2 \pi \psi_{t}\right)
$$

The result of this calculation is the same as in (3) but the number of computations, $\exp \left(i 2 \pi \psi_{t}\right)$, decreases in comparison with the direct computation (3) by a factor of $M / T=2 \pi T$. The computation of the sum (3) is thus reduced to building a histogram $g\left(\psi_{t}\right)$ by $(7)$ and then calculating the sum (8).

Further improvement of the efficiency of the algorithm is achieved using the fact that the terms $\exp \left(i 2 \pi \psi_{t}\right)$ on a uniform grid $\psi_{t}$ can be found by consecutive multiplication of complex numbers. By denoting $E_{t}=\exp \left(i 2 \pi \psi_{t}\right)$, we obtain, instead of (8),

$$
\begin{aligned}
& \sum_{t=1}^{T} g\left(\psi_{t}\right) E_{t}, \quad E_{t}=\Delta E E_{t-1}, \quad E_{0}=1+i 0, \\
& \Delta E=\exp \left(\frac{i 2 \pi}{T}\right),
\end{aligned}
$$

( $E_{0}$ is complex and $E_{0}=1$ ). Therefore, the determination of $U(p)$ is independent of the number $l$; also, it is sufficient to calculate $\Delta E$ only once.

A technique similar to (9) is used to determine the centre of $\Delta S_{m}$. Let us have an $m$-paired index $(k, j), k=1,2, \ldots, k_{n}$, in the region of a ring defined by the radii from $R_{n-1}$ to $R_{n}$, in (3): $k_{n}=\max \left(1,\left(R_{n}-R_{n-1}\right) / \sigma\right)$. Then the step along the radius is $\Delta \rho=\left(R_{n}-R_{n-1}\right) / k_{n}$ and the current radius is $\rho_{k}=$ $(k-0.5) \Delta \rho$. The number $I$ of divisions with respect to angle $\varphi$ is $I=\max \left(4,2 \pi \rho_{k} / \sigma\right)$. If $a_{n j}$ is a complex number whose components are the coordinates of the centre of $\Delta S_{k j}$, then the sequence $a_{k j}$ is found from the relation:

$$
a_{k j}=\Delta a_{k} a_{k, j-1} ; \quad j=1,2, \ldots, I_{k},
$$

where

$$
\Delta a_{k}=\exp \left(\frac{i 2 \pi}{I_{k}}\right) ; \quad a_{k, 0}=\rho_{n}+i 0 .
$$

The algorithm [9] was tested on diffraction on Fresnel zone plate lens in $\mathrm{mm}$ and submm wave. A measure of deviation between the theory and the experimental data did not exceed 5\%.

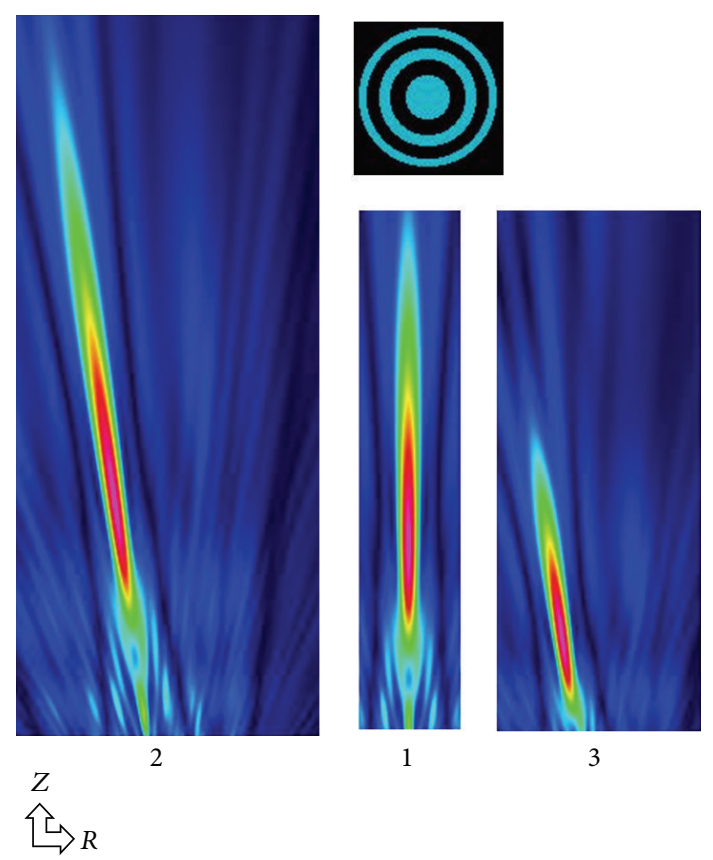

FIGURE 3: The calculation experiment results: Quasi-color images of a Bessel beams: 1-the "normal" condition, 2-the wavelength was changed to $-5 \%$ and the point-like source of radiation was moved up to $D / 2$ from optical axis, 3 -the wavelength was changed to $+5 \%$, and the point-like source of radiation was moved up to $D / 2$ from optical axis. The color is proportional to the field intensity: from blue (low) to red (high).

\section{Scanning Properties of Diffractive "Lens-Plus-Axicon" Lens}

The results of calculation experiment on Bessel beam formation by flat diffractive element are shown in Figure 3. The parameters of a diffractive element were as follows: design wavelength $0.5 \mathrm{~mm}, \gamma=0.157$, diameter of the element $D=$ $15 \mathrm{~mm}$, and the distance to on-axis point-like source $50 \mathrm{~mm}$.

The size of the centre spot is only slightly larger than the wavelength of the beam. The focusing properties of a diffractive "lens-plus-axicon" lens take place both with onand off-axis point-like source of radiation in wide spectrum of wavelength. The observed propagation distance of the Bessel beam depends on actual wavelength. This comparison illustrates clearly the increased depth of field of a Bessel beam. Moreover it has been shown that focusing properties of diffractive "lens-plus-axicon" lens may be used for scan of a 3D space by moving a point-like source [10]. Beams of this type may be useful within 3D imaging applications with high field of view where the short Rayleigh range of a Gaussian beam of similar spot size would lead to a poor depth of field and field of view.

In addition to the binary diffractive element described in this work also several other coding schemes of phase have been suggested in [10]. The higher-order Bessel beams using diffractive "lens-plus-axicon" lens, which generate beam profiles featuring so-called "dark beams" on axis, also have been investigated. The scanning and frequency properties of phase 


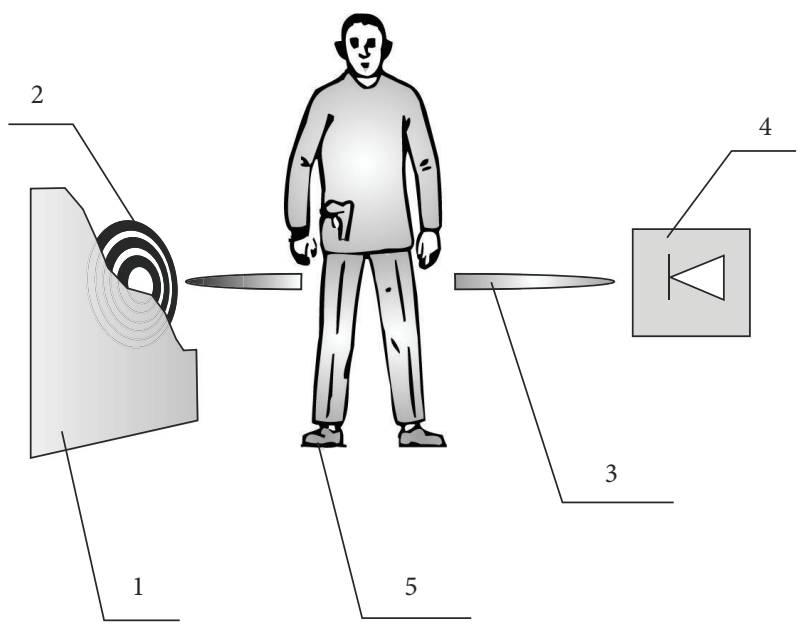

FIGURE 4: Description of $\mathrm{THz}$ security system: 1-the wall, 2diffractive optic (axicon antenna), 3-nondiffractive beam, 4detector, 5-trespasser.

and higher-order Bessel beams using diffractive "lens-plusaxicon" lens were also investigated [10].

\section{State of the Art of Security Scanning System}

Based on investigations described above we may offer the novel concept of the following security system. The system is based on a novel $\mathrm{THz}$ diffractive optics for scanning the person (without the application of THz laser) and on a sensitive scheme for the detection of the reflected and scattered $\mathrm{THz}$ radiation (Figure 4). Novel security system working similar to a laser security system: a THz/MMW source is a concentrated $\mathrm{MMW} / \mathrm{THz}$ source that puts out a straight line "pencil beam" THz/MMW. The imaging systems rely on advanced MMW/THz technology [6]. The development of enabling technology, namely, sensitive detector arrays and $\mathrm{MMW} / \mathrm{THz}$ diffractive optics, will allow to build compact, easy-to-use MMW/THz imaging systems without expensive cost $\mathrm{THz}$ laser. Also miniaturized $\mathrm{THz}$ sources with sufficient output power, uncooled 2-dimensional detector arrays for real-time data acquisition, and high-efficient $\mathrm{THz}$ optics are fundamental components for handheld $\mathrm{THz}$ illumination and imaging systems.

\section{References}

[1] Richard Gaughan. How Do Laser Security Systems Work?, http://science.opposingviews.com/laser-security-systemswork-1631.html.

[2] K. Su, L. Moeller, R. B. Barat, and J. F. Federici, "Experimental comparison of terahertz and infrared data signal attenuation in dust clouds," Journal of the Optical Society of America A, vol. 29, no. 11, pp. 2360-2366, 2012.

[3] L. Yun-Shik, Principles of Terahertz Science and Technology, Springer, 2008.

[4] R. M. Herman and T. A. Wiggins, "Production and uses of diffractionless beams," Journal of the Optical Society of America A, vol. 8, pp. 932-942, 1991.
[5] I. A. Mikhaltsova, V. I. Nalivaiko, and I. S. Soldatenkov, "Kinoform axicons," Optik, vol. 67, no. 3, pp. 267-278, 1984.

[6] I. V. Minin and O. V. Minin, "Active millimeter/THz waves high-quality 3D imaging for scientific, medical and homeland security applications," in Proceeding of an International Joint Conference of 4th ESA Workshop on Millimeter Wave Technology and Applications, The 8th Topical Symposium on Millimeter Waves (TSMMW '06,) The 7th MINT Millimeter Wave International Symposium (MINTMIS '06), pp. 131-136, MilliLab, February 2006.

[7] M. Born and E. Wolf, Principles of Optics, Cambridge University Press, 7th edition, 1999.

[8] F. X. Baibulatov, I. V. Minin, and O. V. Minin, "Investigation of the focusing properties of the Fresnel zonel plate," Journal of Communications Technology and Electronics, vol. 30, no. 9, pp. 1034-1039, 1985.

[9] O. V. Minin and I. V. Minin, Diffractional Optics of Millimeter Waves, IOP Publisher, Boston, Mass, USA, 2004.

[10] I. V. Minin, "Scanning properties of the diffractive "lensplus-axicon" lens in THz," in Proceedings of the 11th Microcoll Symposium, pp. 233-236, Budapest, Hungary, September 2003. 

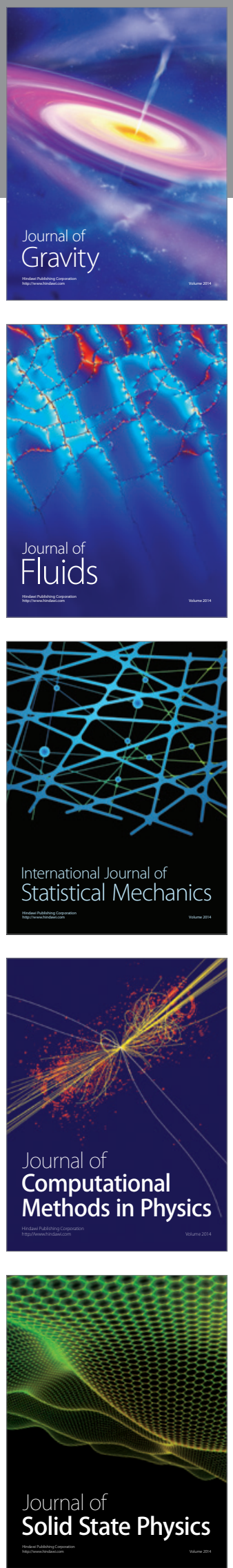

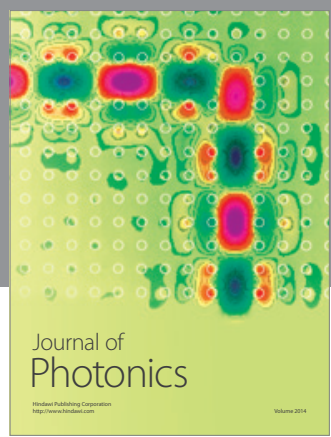

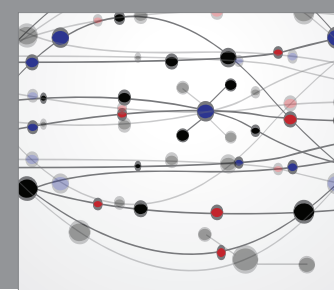

The Scientific World Journal

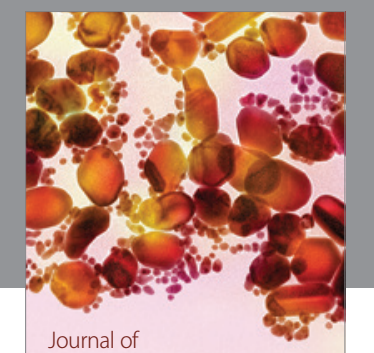

Soft Matter
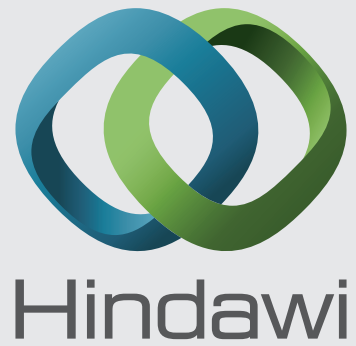

Submit your manuscripts at

http://www.hindawi.com
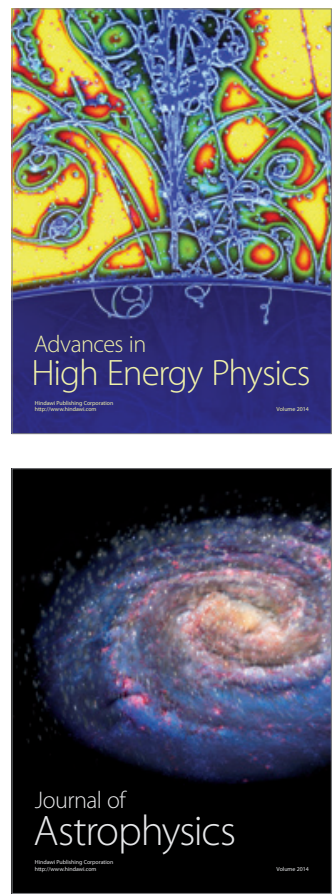
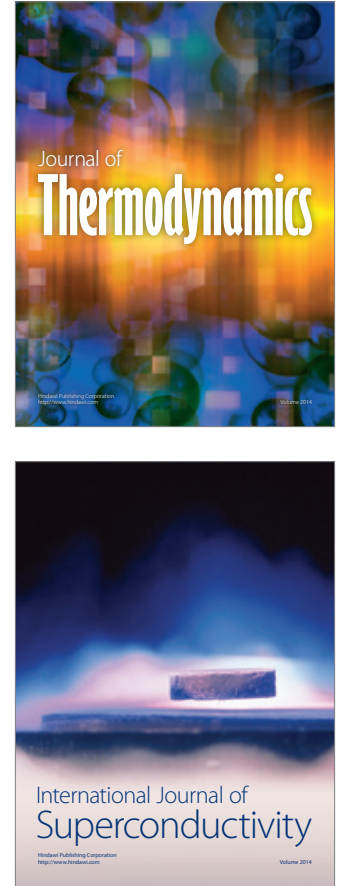
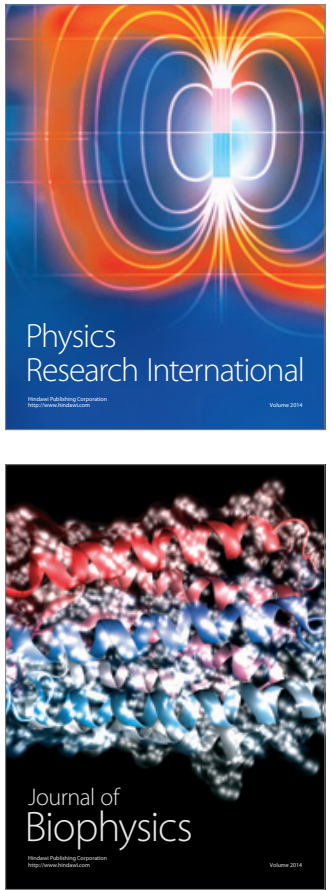
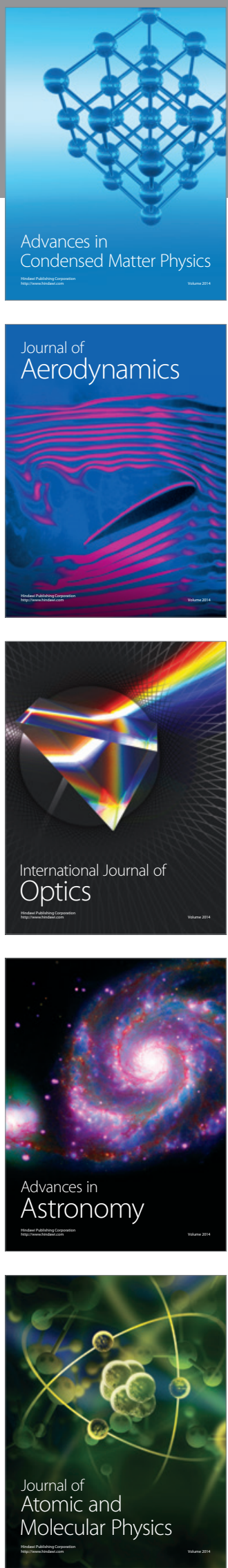